\title{
X-ray/UV campaign on the Mrk 279 outflow: Density diagnostics in Active Galactic Nuclei using O v K-shell absorption lines
}

\author{
J. S. Kaastra ${ }^{1}$, A. J. J. Raassen ${ }^{1,2}$, R. Mewe ${ }^{1, \dagger \star}$, N. $\operatorname{Arav}^{3}$, E. Behar ${ }^{4}$, E. Costantini ${ }^{1}$, J. R. Gabel ${ }^{3}$, G. A. Kriss ${ }^{5}$, \\ D. Proga ${ }^{3}$, M. Sako ${ }^{6}$, and K. C. Steenbrugge ${ }^{1}$
}

1 SRON National Institute for Space Research Sorbonnelaan 2, 3584 CA Utrecht, The Netherlands e-mail: J.Kaastra@sron.nl

2 Astronomical Institute "Anton Pannekoek", Kruislaan 403, 1098 SJ Amsterdam, The Netherlands

3 CASA, University of Colorado, 389 UCB, Boulder, CO 80309-0389, USA

${ }^{4}$ Department of Physics, Technion-Israel Institute of Technology, Haifa 32000, Israel

5 Space Telescope Science Institute, 3700 San Martin Drive, Baltimore, MD 21218, USA

${ }^{6}$ KIPAC/SLAC, 2575 Sand Hill Road M/S 29, Menlo Park, Ca 94025, USA

Received 8 June 2004 / Accepted 12 August 2004

\begin{abstract}
One of the main problems in modeling the ionised outflows in Active Galactic Nuclei is the unknown distance of the outflowing wind to the central source. Only if the density is known this distance can be determined through the ionisation parameter. Here we study density diagnostics based upon O v transitions. O v is known to have metastable levels that are density dependent. We study the population of those levels under photoionisation equilibrium conditions and determine for which parameter range they can have a significant population. We find that resonance line trapping plays an important role in reducing the critical densities above which the metastable population becomes important. We investigate the K-shell absorption lines from these metastable levels. Provided that there is a sufficient population of the metastable levels, the corresponding K-shell absorption lines are detectable and are well separated from the main absorption line originating from the ground state. We then present the Chandra LETGS spectrum of the Seyfert 1 galaxy Mrk 279 that may show for the first time the presence of these metastable level absorption lines. A firm identification is not yet possible due to both uncertainties in the observed wavelength of the strongest line as well as uncertainties in the predicted wavelength. If the line is indeed due to absorption from O v, then we deduce a distance to the central source of one light week to a few light months, depending upon the importance of additional heating processes.
\end{abstract}

Key words. galaxies: individual: Mrk 279 - galaxies: Seyfert - galaxies: quasars: absorption lines - X-rays: galaxies

\section{Introduction}

Active Galactic Nuclei (AGN) have X-ray spectra that are dominated by nonthermal emission from the immediate surroundings of a supermassive black hole. Seyfert galaxies constitute the lower luminosity class of AGN. In a significant fraction of all Seyfert galaxies the spectral signatures of the so-called warm absorber are visible (e.g., Reynolds 1997; George et al. 1998). This warm absorber is very likely a photoionised outflow from the accretion disk, with strong UV (for example Crenshaw et al. 1999) and X-ray absorption lines (Kaastra et al. 2000). X-ray observations with high sensitivity show the presence of absorption lines with a broad range of ionisation parameter $\xi=L / n r^{2}$, where $L$ is the 1-1000 Ryd luminosity, $n$ the density and $r$ the distance to the central source. A good example of this broad range in ionisation parameter is the XMMNewton Reflection Grating Spectrometer (RGS) spectrum of NGC 5548, which shows the presence of K-shell absorption

* Deceased 4 May 2004. lines from all oxygen ions between O III-O VIII (Steenbrugge et al. 2003).

One of the main problems in modeling this outflow is that its distance $r$ from the nucleus is hard to measure. Photoionisation modeling only yields the product $n r^{2}$, as well as the column density $n d$ with $d$ the thickness of the absorbing layer in the line of sight. Lacking a density, both the thickness $d$ and the distance $r$ are essentially unknown. Gabel et al. (2003) detected UV absorption lines from the metastable 2s $2 \mathrm{p}{ }^{3} \mathrm{P}$ triplet in $\mathrm{C}$ III around $1175 \AA$ in the highest velocity outflow component in NGC 3783. These measurements indicate a relatively high density of order $10^{15} \mathrm{~m}^{-3}$ corresponding to an upper limit of the distance to the nucleus of $0.3 \mathrm{pc}$. However these density estimates are somewhat uncertain due to the contamination of the C III resonance line at $977 \AA$ with Galactic absorption in that source. Behar et al. 2003 pointed out that the $2 \mathrm{~s} 2 \mathrm{p}$ triplet of C III can in fact be populated at low densities. Only one fine-structure level in the triplet requires high densities (see, e.g., Fig. 1 in Bhatia \& Kastner 1993). Indeed 
updated calculations by Gabel et al. (2004a) show a lower density of $3 \times 10^{10} \mathrm{~m}^{-3}$ and a distance of $25 \mathrm{pc}$.

Other constraints on density hence distance may be derived from reverberation studies of the warm absorber in response to continuum variations, by measuring the recombination time scale. Long grating observations of NGC 3783 with XMM-Newton (Behar et al. 2003) and with Chandra (Netzer et al. 2003) have allowed for attempts to study the response of the absorber to the ionising continuum through reverberation. However, since no response was detected these could provide only lower limits (of the order of $0.5 \mathrm{pc}$ ) to the distance of the absorber from the central source. Reeves et al. (2004) did claim to see variability in the $\mathrm{Fe}-\mathrm{K}$ absorption of that source, albeit with CCD spectra. They deduce an upper limit to the distance of the absorber to the central source of $0.02 \mathrm{pc}$.

In stellar coronae, with collisional ionisation equilibrium, several X-ray emission lines are density-dependent, for example the forbidden and intercombination lines of the O VII triplet. As these lines have low oscillator strengths, it is impossible to observe them in absorption. In some isoelectronic sequences, close above the ground state of the ion there exist metastable levels that can have a significant population. A well known example is the Be-sequence, where the $\mathrm{C}$ III line at $1909 \AA$ is often used as a density diagnostic in plasmas. Another ion in this iso-electronic sequence is $\mathrm{OV}$, where the metastable $1 \mathrm{~s}^{2} 2 \mathrm{~s} 2 \mathrm{p}{ }^{3} \mathrm{P}$ level is only $10 \mathrm{eV}$ above the ground state $1 \mathrm{~s}^{2} 2 \mathrm{~s}^{2}{ }^{1} \mathrm{~S}_{0}$. The $J=0$ term of this triplet cannot decay radiatively to the ground state, leading to a significant population of this level at all densities. K-shell absorption from the ground state of this ion (at $22.3 \AA$ ) has been observed in AGN (NGC 5548, Steenbrugge et al. 2003, 2004; NGC 4051, Ogle et al. 2004); and recently Mrk 279 (Costantini et al. 2004). In this last source the spectrum indicates the possible presence of absorption from the metastable level of $\mathrm{OV}$. The critical density for the population of this metastable level is around $10^{16} \mathrm{~m}^{-3}$, a relevant density for AGN outflows. The presence or absence of K-shell absorption lines from the metastable level of $\mathrm{OV}$ then is an important density diagnostic, which can serve to constrain distance and geometry of the outflow.

In principle transitions from the metastable level of $\mathrm{OV}$ can also be studied using the UV absorption lines from the 2 s $2 p$ triplet to $2 p^{2}$ triplet near $760 \AA$ A. Pettini \& Boksenberg (1986) identified these lines in IUE spectra of the BAL quasar PG 0946+301. However, in HST data of the same object no evidence for these lines was found (Arav et al. 1999). But in yet another BAL quasar (QSO B0226-1024) Korista et al. (1992) found possible evidence for the presence of these lines, implying densities of the order of $10^{17} \mathrm{~m}^{-3}$. However, due to Galactic absorption such an analysis can only be done for bright, highly redshifted quasars. For nearby Seyfert galaxies K-shell X-ray absorption lines are the ideal tool to study densities.

In this paper we study the expected population of the metastable level of $\mathrm{OV}$ under photoionised conditions, as well as the wavelengths and oscillator strengths of the corresponding K-shell absorption lines. We then present a possible detection of absorption from $\mathrm{OV}^{*}$ levels in the Chandra LETGS spectrum of Mrk 279.
Table 1. Energy levels of O v. Energies are taken from Wiese et al. (1996). Levels 2-4 are the metastable levels discussed in this paper.

\begin{tabular}{rrrr}
\hline \hline Level & Configuration & $J$ & Energy $(\mathrm{eV})$ \\
\hline 1 & $2 \mathrm{~s}^{2}{ }^{1} \mathrm{~S}$ & 0 & 0 \\
2 & $2 \mathrm{~s} 2 \mathrm{p}{ }^{3} \mathrm{P}$ & 0 & 10.16 \\
3 & & 1 & 10.18 \\
4 & & 2 & 10.21 \\
5 & $2 \mathrm{~s} 2 \mathrm{p}{ }^{1} \mathrm{P}$ & 1 & 19.69 \\
6 & $2 \mathrm{p}^{2}{ }^{3} \mathrm{P}$ & 0 & 26.47 \\
7 & & 1 & 26.49 \\
8 & & 2 & 26.52 \\
9 & $2 \mathrm{p}^{2}{ }^{1} \mathrm{D}$ & 2 & 28.73 \\
10 & $2 \mathrm{p}^{2}{ }^{1} \mathrm{~S}$ & 0 & 35.70 \\
\hline
\end{tabular}

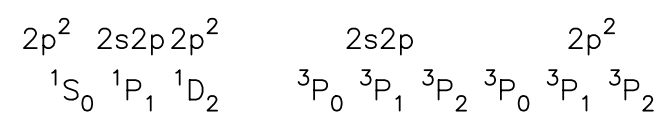

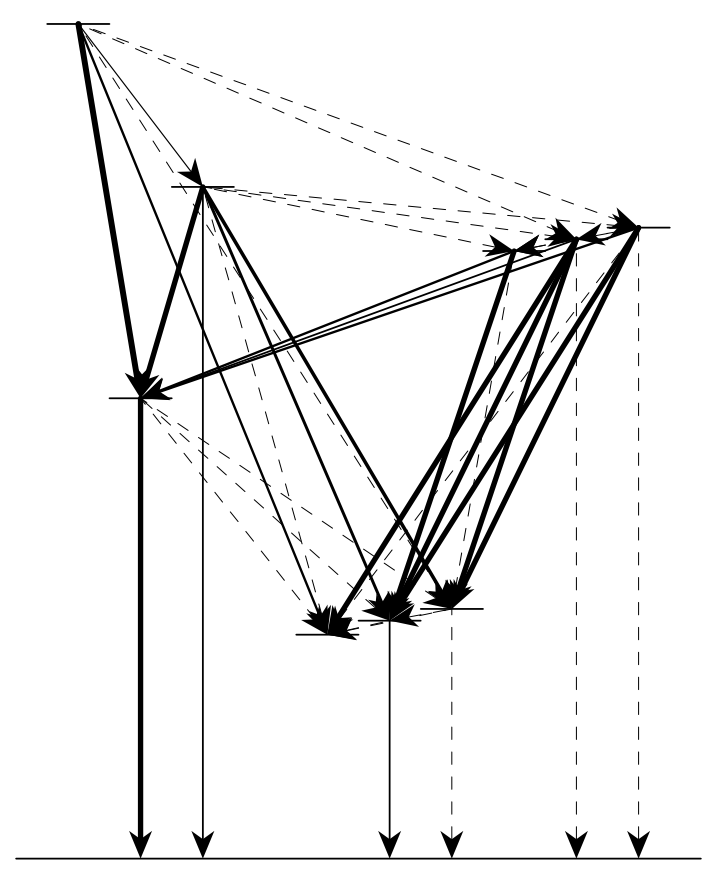

Fig. 1. Energy level diagram of O v. Only the $n=2$ levels are shown. The energy differences within the ${ }^{3} \mathrm{P}$ triplets have been exaggerated for clarity. Solid lines: transitions with transition probabilities larger than $1 \mathrm{~s}^{-1}$; the line thickness is proportional to the logarithm of the transition probability. Dashed lines: transitions with transition probabilities less than $1 \mathrm{~s}^{-1}$.

\section{Level populations}

\subsection{Transition rates}

We made a model for the population of all 10 levels with principal quantum number $n=2$. These levels are listed in Table 1 . The energy-level diagram is shown in Fig. 1. We omit the $n>2$ levels from our calculation, as the lowest of these levels has an energy of $67.82 \mathrm{eV}$. This is too high as compared to the typical temperatures of a few $\mathrm{eV}$ for which $\mathrm{OV}$ is formed under photoionised conditions. 
The population levels $n_{i}$ were solved using the equation $R_{i j} n_{j}=b_{i}$,

where we sum over $j$ and $R_{i j}$ is the total transition rate matrix from level $j$ to level $i$, and $b_{i}$ represents source/loss terms like ionisation from $\mathrm{O} I \mathrm{~V}$ to $\mathrm{O} \mathrm{V}$ or recombination from $\mathrm{O}$ VI to $\mathrm{O} \mathrm{V}$. Other loss terms like ionisation or recombination of $\mathrm{O} \mathrm{V}$ itself can be accomodated in $R_{i j}$, as these processes are proportional to the $\mathrm{O} v$ density.

Radiative transition rates between the levels of Table 1 were taken from the compilation of Wiese et al. (1996).

We took the excitation rates from Safranova et al. (1995) for the following transitions: $2 s^{2}-2 s-p$ and $2 s-2 p-2 p^{2}$. The remaining rates were taken from Kato et al. (1990). Kato et al. give only the total rates for the following transitions involving a singlet and a triplet: $2 \mathrm{~s}^{2}-2 \mathrm{p}^{2}{ }^{3} \mathrm{P}, 2 \mathrm{~s} 2 \mathrm{p}^{3} \mathrm{P}-2 \mathrm{~s} 2 \mathrm{p}{ }^{1} \mathrm{P}, 2 \mathrm{p}^{2}{ }^{3} \mathrm{P}-2 \mathrm{p}^{2}{ }^{1} \mathrm{D}$ and $2 p^{2}{ }^{3} \mathrm{P}-2 \mathrm{p}^{2}{ }^{1} \mathrm{~S}$. Lacking more information, we therefore subdivided these rates according to the statistical weights of the triplets involved. This is also found to be valid for the distorted wave calculations of Zhang \& Sampson (1992), which explicitly give multiplet-resolved rates. There is a caveat, however. As noted by Safranova et al. (1995) in the forbidden transitions $\left(J^{\prime}-J=0-0,0-2,2-0\right)$, only the exchange contribution to the collision strength is important, and the direct part is zero. The ${ }^{3} \mathrm{P}_{0}-{ }^{1} \mathrm{D}_{2},{ }^{3} \mathrm{P}_{0}-{ }^{1} \mathrm{~S}_{0}$ and ${ }^{3} \mathrm{P}_{2}-{ }^{1} \mathrm{~S}_{0}$ transitions of the above mentioned configurations are forbidden and would suffer from this effect. As however in most astrophysical situations the occupation of the $2 \mathrm{p}^{2}$ levels is low, and these levels have strong radiative transitions to lower levels, the errors made by our approximation are not too serious.

We added the proton excitation rates within the $2 \mathrm{~s} 2 \mathrm{p}$ and $2 \mathrm{p}^{2}{ }^{3} \mathrm{P}$ triplets from Doyle et al. (1980).

We also included radiative recombination (OVI to $\mathrm{OV}$ and $\mathrm{OV}$ to $\mathrm{OIV}$ ) and collisional ionisation rates (O IV to $\mathrm{OV}$ and $\mathrm{OV}$ to $\mathrm{OVI}$ ). We took the total radiative recombination rates from Arnaud \& Rothenflug (1985) and assume that the rates towards the $n=2$ levels, either direct or indirect, are proportional to the statistical weight of these levels. The collisional ionisation rates were also taken from Arnaud \& Rothenflug (1985). For ionisation of OIV, we assume that all O IV ions are in the $2 \mathrm{~s}^{2} 2 \mathrm{p}{ }^{2} \mathrm{P}$ ground state, and that $2 \mathrm{~s}$-shell ionisation of $\mathrm{O}$ IV results into a $2 \mathrm{~s} 2 \mathrm{p}{ }^{3} \mathrm{P}$ state of $\mathrm{OV}$ with the population of the sublevels distributed according to their statistical weight. Note however that for the parameter range that we consider the collisional ionisation and radiative recombination rates are relatively unimportant.

At low temperatures charge transfer reactions with neutral hydrogen become important. We included the charge transfer recombination rates for recombination of $\mathrm{O} V \mathrm{VI}$ and $\mathrm{OV}$ from Rakovic et al. (2001). Similar to the radiative recombination, we assume that the rates towards the $n=2$ levels, either direct or indirect, are proportional to the statistical weight of these levels.

\subsection{Collisional ionisation equilibrium}

Using the rates determined in the previous section, we determine equilibrium population levels for a range of densities

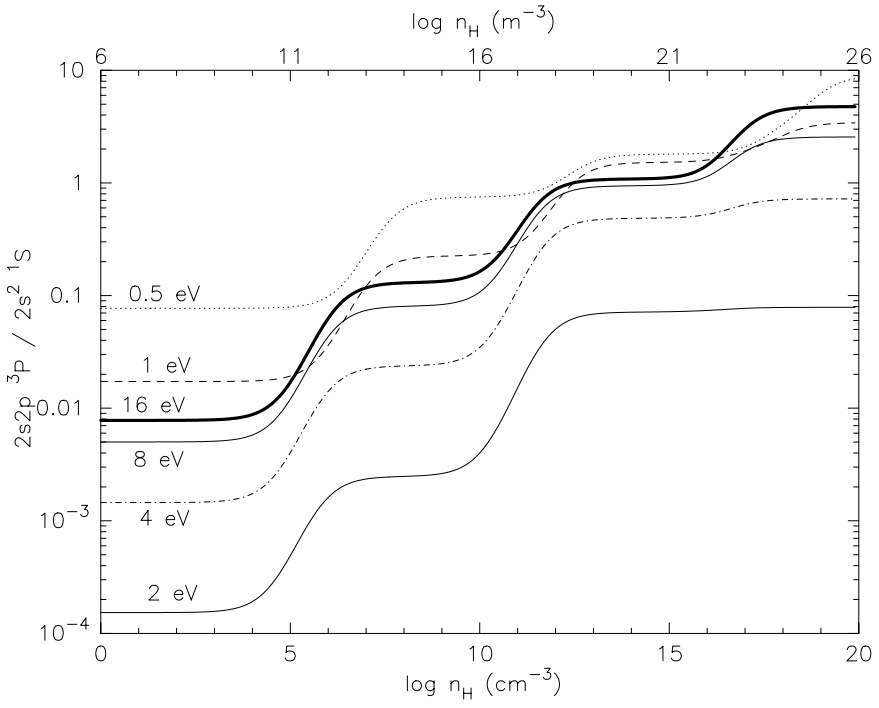

Fig. 2. Population of the $2 s 2 p{ }^{3} \mathrm{P}$ triplet with respect to the ground state in $\mathrm{OV}$ as a function of density and $k T$ (in eV) as labeled. All three sublevels $(J=0, J=1$ and $J=2)$ have been added.

and temperatures, first under collisional ionisation equilibrium (CIE) conditions. We restrict the temperature range to $k T<20 \mathrm{eV}$; higher temperatures are not expected under photoionised conditions, and moreover they would result in a significant amount of excitation to the $n=3$ levels, with the corresponding downwards radiative decays.

For this temperature range, the effect of collisional ionisation is negligible, as the electrons have insufficient energy to overcome the $113.896 \mathrm{eV}$ ionisation potential. Radiative recombination affects the population of the $2 \mathrm{~s} 2 \mathrm{p}{ }^{3} \mathrm{P}$ triplet by no more than $3 \%$. Proton excitation modifies the populations only significantly (more than 3\%) for temperatures higher than $10 \mathrm{eV}$, but even for $k T=30 \mathrm{eV}$ the effects are smaller than $11 \%$ for all densities.

Charge transfer recombination due to collisions with $\mathrm{HI}$ atoms appears to be very important at temperatures below $2 \mathrm{eV}$. This is mainly due to the large recombination cross section at low temperatures and the high neutral fraction at these low temperatures. Since under CIE conditions the O V fraction is less than 0.001 for temperatures below $9 \mathrm{eV}$, inclusion of charge transfer recombination is of little practical use under those circumstances, however.

Figure 2 shows the relative occupation $P$ of the $2 \mathrm{~s} 2 \mathrm{p}{ }^{3} \mathrm{P}$ triplet with respect to the ground state. The enhancement of this ratio at $k T=0.5 \mathrm{eV}$ and $k T=1 \mathrm{eV}$ is due to the charge transfer recombinations. The sharp increases in $P$ as a function of hydrogen density $n_{\mathrm{H}}$ around densities of a) $10^{12}$, b) $10^{17}$ and c) $10^{22} \mathrm{~m}^{-3}$ are due to the following. At a), the collisional coupling of the $2 \mathrm{~s} 2 \mathrm{p}^{3} \mathrm{P}_{2}$ level with the $2 \mathrm{~s} 2 \mathrm{p}^{3} \mathrm{P}_{1}$ level (either direct or via excitation to the $2 \mathrm{p}^{2}{ }^{3} \mathrm{P}_{1}$ level followed by radiative decay) becomes stronger than the radiative decay by a forbidden transition to the ground state. At $b$ ), the collisional coupling of the $2 \mathrm{~s} 2 \mathrm{p}{ }^{3} \mathrm{P}_{1}$ level with the other triplet levels is stronger than its radiative decay to the ground state. Finally, at c) the ground state depopulates due to strong collisional coupling of the $2 \mathrm{~s} 2 \mathrm{p}{ }^{1} \mathrm{P}$ level with the $2 \mathrm{p}^{2}{ }^{1} \mathrm{D},{ }^{1} \mathrm{~S}$ levels. This causes the 


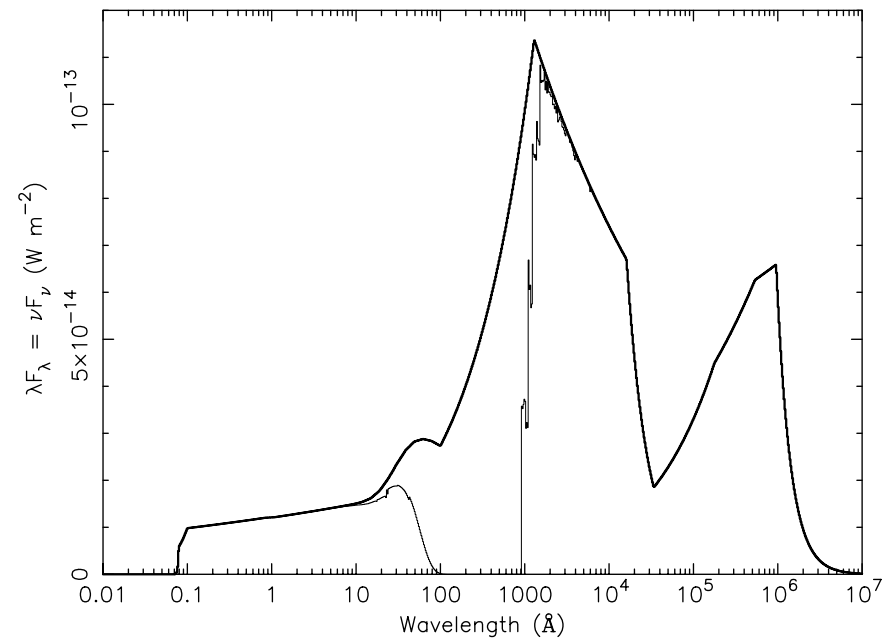

Fig. 3. Spectral energy distribution (thick line) used for our photoionisation calculations. The thin line shows the same spectrum absorbed by a Galactic foreground of cold gas with a hydrogen column density of $1.64 \times 10^{24} \mathrm{~m}^{-2}$ and $\sigma_{v}=10 \mathrm{~km} \mathrm{~s}^{-1}$.

ratio of the triplet with respect to the ground state to increase (the ratio with respect to the sum of all $\mathrm{OV}$ levels does not change).

\subsection{Photoionisation equilibrium}

For photoionised plasmas we use the same approach as before, only the transition matrix $R_{i j}$ and the source term $b_{i}$ contains more terms. We now include both photo-excitation and stimulated emission between the $n=2$ levels. As we will use an ionising spectrum with a strong UV component (see below), photo-excitations towards higher levels than $n=2$ are relatively unimportant. We include photoionisation of O IV from both the $2 \mathrm{p}$ and $2 \mathrm{~s}$ shells. For our ionising spectrum, the photoionisation rate of O IV from the $1 \mathrm{~s}$ shell is only $2.5 \%$ of the $2 \mathrm{p}$ rate, and as $1 \mathrm{~s}$ ionisation of O IV is followed in most cases by an Auger transition, producing O VI, we neglect this process here. We also neglect the similar process of K-shell ionisation of O III followed by an Auger transition, as we assume that the concentration of $\mathrm{O}$ III relative to $\mathrm{O} \mathrm{V}$ may be small and again $\mathrm{K}$-shell photoionisation is rare relative to L-shell photoionisation for our ionising spectrum. However, we included the 5\% contribution of $1 \mathrm{~s}$ photoionisation of $\mathrm{O} \mathrm{V}$ to the total $n=2$ photoionisation rate, as this represents a loss term for which we are not interested in the end product.

For the photoionising spectrum we adopt a model based upon the observed Chandra and UV continuum of Mrk 279 (Costantini et al. 2004). This model is similar to the model employed for NGC 5548 by Kaastra et al. (2002). The spectrum contains a strong UV bump (Fig. 3 ). We normalized it to a $1-1000$ Ryd luminosity $L$ of $8.31 \times 10^{37} \mathrm{~W}$, in order to reproduce the observed UV and X-ray continuum of Mrk 279. Note that for this spectral shape the relation between the photoionisation parameters $\xi$ and $U$ is given by $U=Q / 4 \pi r^{2} n c=$ $0.036 \xi$, where $Q$ is the number of hydrogen ionising photons and $\xi$ is expressed in units of $10^{-9} \mathrm{~W} \mathrm{~m}$. The concentrations of O IV-O VI as well as the temperature $T(\xi)$ as a function of the

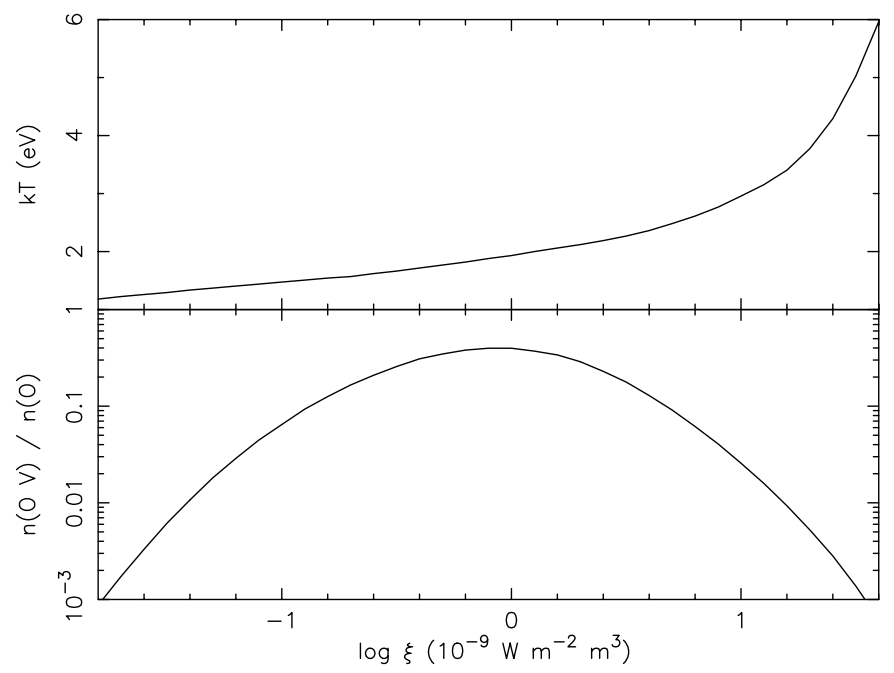

Fig. 4. Temperature (upper panel) and $\mathrm{O} v$ fraction (lower panel) as a function of ionisation parameter $\xi$. The maximum $\mathrm{OV}$ concentration of $40 \%$ occurs for $\log \xi=-0.05$, at a temperature $k T=1.9 \mathrm{eV}$ (to be compared with $47 \%$ at $k T=21.0 \mathrm{eV}$ under CIE conditions).

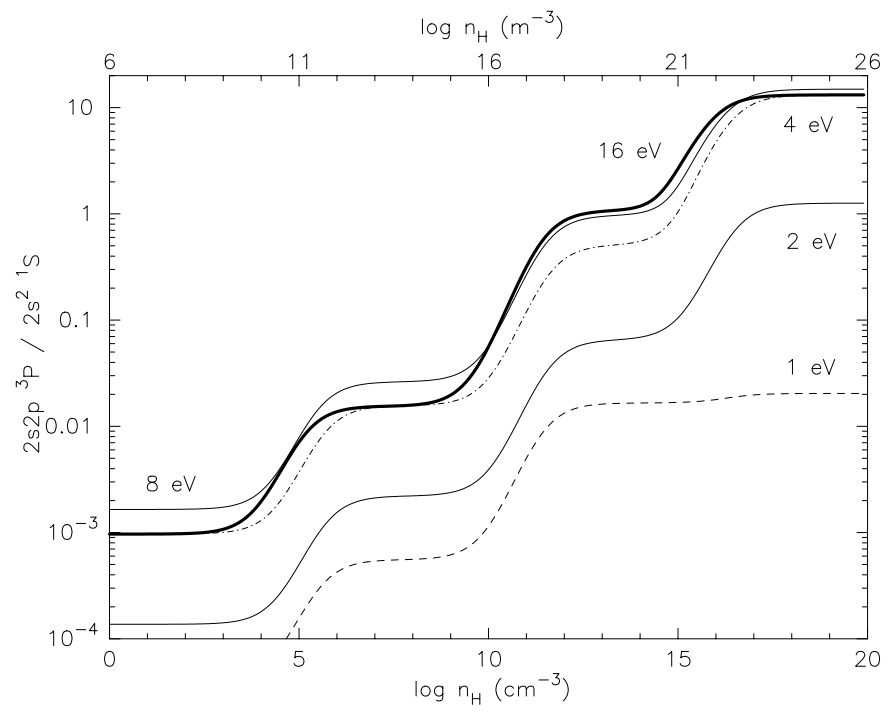

Fig. 5. Population of the $2 \mathrm{~s} 2 \mathrm{p}{ }^{3} \mathrm{P}$ triplet with respect to the ground state in $\mathrm{OV}$ as a function of density and $k T$ (in $\mathrm{eV}$ ) as labeled for a photoionised plasma. All three sublevels $(J=0, J=1$ and $J=2)$ have been added.

ionisation parameter $\xi$ were obtained from a set of runs with Cloudy Version 95.06 (Ferland 2002) for a thin photoionised slab at low density and with solar abundances. In that approximation, $T(\xi)$ and the ion concentrations only depend upon the shape of the ionizing spectrum. Figure 4 shows the OV concentration and $T(\xi)$ relation. We then determined the level populations as a function of $n_{\mathrm{H}}$ and $T$; inverting $T(\xi)$ yields $\xi$ and using $\xi, L$ and $n_{\mathrm{H}}$ we then know the distance of the absorber to the central source and hence the relevant fluxes for photoionisation and photo-excitation.

We show our results in Fig. 5. At low temperatures $(k T<$ $1 \mathrm{eV}$ ) the neutral hydrogen fraction is now much smaller than in a collisional equilibrium plasma, resulting in less important effects of charge transfer recombination. For temperatures $k T$ 
between $2-4 \mathrm{eV}$ and densities below $10^{20} \mathrm{~m}^{-3}$ the results for photoionisation equilibrium (PIE) and CIE are not significantly different. At higher densities photo-excitation of in particular the $2 s^{2}-2 s 2 p{ }^{1} P_{1}$ resonance line $(629.73 \AA)$ is more important than collisional excitation from the ground state to the $2 \mathrm{~s} 2 \mathrm{p}^{1} \mathrm{P}_{1}$ level, causing enhanced higher level populations.

\subsection{Resonance line trapping}

In Sect. 2.3, our treatment of PIE assumed that $\mathrm{OV}$ is located in a photoionised slab with negligible column density. However modeling of the Mrk 279 spectrum shows that O V has a column density of order $10^{20} \mathrm{~m}^{-2}$. For a velocity broadening $\sigma_{v}$ of $50 \mathrm{~km} \mathrm{~s}^{-1}$ this implies that the $2 \mathrm{~s}^{2}-2 \mathrm{~s} 2 \mathrm{sp}{ }^{1} \mathrm{P}_{1}$ resonance line has an optical depth of 69 at the line center. As for a broad range of parameter space radiative decay through this emission line is the dominant decay proces of the upper level $j$, line photons effectively undergo resonance line scattering. The direct escape probability $P_{\text {esc }}$ per emitted photon is therefore very small, and this leads effectively to a smaller radiative decay rate $A_{i j}^{\prime}=P_{\text {esc }} A_{i j}$, with $A_{i j}$ the true transition probability. We follow here the approach of Hollenbach \& McKee (1979) and Kallman \& McCray (1982) in order to approximate $P_{\text {esc }}$ for a slab:

$$
P_{\mathrm{esc}}=\frac{\left(A_{i j} / A_{\mathrm{tot}}\right)}{1 .+\tau_{0} \sqrt{2 \pi \ln \left(2.13+\tau_{0}^{2}\right)}},
$$

where $\tau_{0}$ is the optical depth at line center, and $A_{\text {tot }}$ is the total transition probability from level $j$ to any higher or lower level, including radiative and collisional processes. Resonance line trapping appears to be relevant for the transition between levels $1-5(629.73 \AA$ ) mentioned above, as well as for the 3-6 (761.13 $\AA), 5-9(1371.30 \AA)$ and 5-10 (774.52 $\AA)$ transitions whenever the $2 \mathrm{~s} 2 \mathrm{p}$ configuration has a significant population.

In Fig. 6 we show the results for our calculations including resonance line trapping. It is seen that the critical density at which the $2 s 2 p$ triplet level gets a significant population can shift downwards by several orders of magnitude for sufficiently large column densities.

\section{X-ray absorption lines}

We have calculated wavelengths and oscillator strengths for all transitions between levels with a K-shell vacancy $\left(1 \mathrm{~s} 2 \mathrm{~s}^{2} 2 \mathrm{p}\right.$, $1 \mathrm{~s} 2 \mathrm{~s} 2 \mathrm{p}^{2}$ and $\left.1 \mathrm{~s}^{2} \mathrm{p}^{3}\right)$ and the $1 \mathrm{~s}^{2} 2 \mathrm{~s}^{2}, 1 \mathrm{~s}^{2} 2 \mathrm{~s} 2 \mathrm{p}, 1 \mathrm{~s}^{2} 2 \mathrm{p}^{2}$ and $1 s^{2} 2 s n p$ levels. We used the code of Cowan (1981) for this purpose.

Table 2 lists the calculated wavelenghts and oscillator strengths $f$ of all lines with $f>0.001$ and principal quantum number $n<10$. We determined only total oscillator strengths and average wavelengths of the multiplets, as the current X-ray instrumentation has insufficient resolution to resolve the individual lines of the multiplets.

An important question is the accuracy of the wavelengths. In general these wavelengths have never been measured in the laboratory, with the exception of the resonance transition A2. This wavelength is $22.374 \pm 0.003 \AA$ (Schmidt et al. 2004).

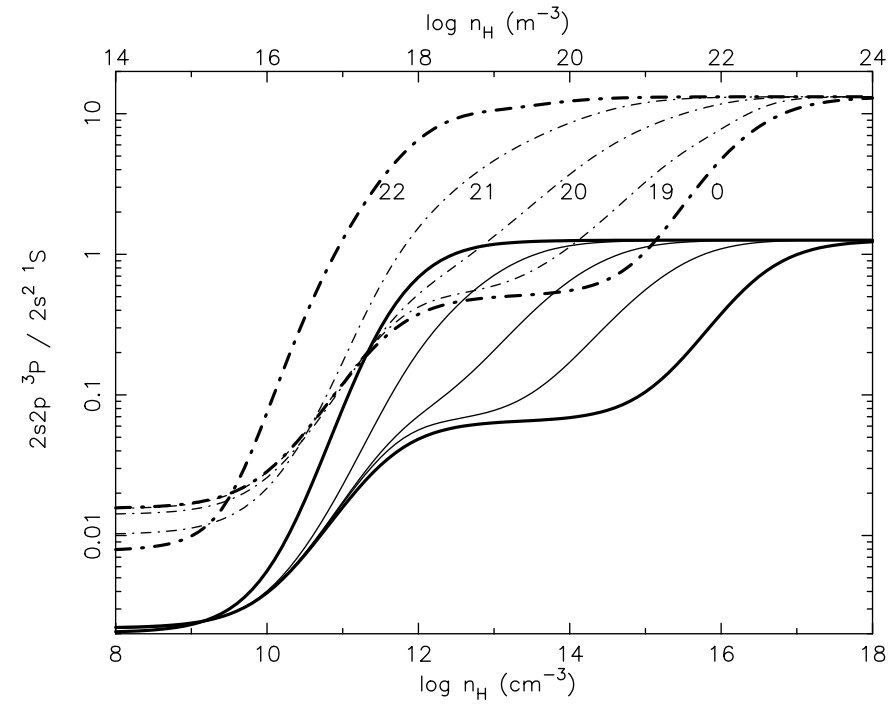

Fig. 6. Population of the $2 \mathrm{~s} 2 \mathrm{p}{ }^{3} \mathrm{P}$ triplet with respect to the ground state in $\mathrm{OV}$ as a function of density and $k T$ (in $\mathrm{eV}$ ) in a photoionised plasma. Solid curves are for $k T=2 \mathrm{eV}$, dash-dotted curves for $k T=$ $4 \mathrm{eV}$. Labels indicate $\log N$ with $N$ the column density of $\mathrm{OV}$ in $\mathrm{m}^{-2}$. All three sublevels ( $J=0, J=1$ and $J=2)$ have been added.

Table 2. Strongest X-ray lines of O v. The values listed apply for the full multiplets (not splitted into sublevels).

\begin{tabular}{|c|c|c|c|c|c|c|}
\hline \multirow[t]{2}{*}{ Label } & \multicolumn{2}{|c|}{ Initial state } & \multicolumn{2}{|c|}{ Final state } & \multirow[t]{2}{*}{$f$} & \multirow{2}{*}{$\begin{array}{c}\lambda \\
(\AA)\end{array}$} \\
\hline & Conf. & Term. & Conf. & Term. & & \\
\hline A2 & $2 s^{2}$ & ${ }^{1} \mathrm{~S}$ & $1 \mathrm{~s} 2 \mathrm{~s}^{2} 2 \mathrm{p}$ & ${ }^{1} \mathrm{P}$ & 0.649 & 22.381 \\
\hline A3 & $2 s^{2}$ & ${ }^{1} \mathrm{~S}$ & $1 \mathrm{~s} 2 \mathrm{~s}^{2} 3 \mathrm{p}$ & ${ }^{1} \mathrm{P}$ & 0.108 & 19.871 \\
\hline A4 & $2 s^{2}$ & ${ }^{1} \mathrm{~S}$ & $1 \mathrm{~s} 2 \mathrm{~s}^{2} 4 \mathrm{p}$ & ${ }^{1} \mathrm{P}$ & 0.041 & 19.258 \\
\hline A5 & $2 s^{2}$ & ${ }^{1} \mathrm{~S}$ & $1 \mathrm{~s} 2 \mathrm{~s}^{2} 5 \mathrm{p}$ & ${ }^{1} \mathrm{P}$ & 0.020 & 19.002 \\
\hline A6 & $2 s^{2}$ & ${ }^{1} \mathrm{~S}$ & $1 \mathrm{~s} 2 \mathrm{~s}^{2} 6 \mathrm{p}$ & ${ }^{1} \mathrm{P}$ & 0.012 & 18.869 \\
\hline A7 & $2 s^{2}$ & ${ }^{1} \mathrm{~S}$ & $1 \mathrm{~s} 2 \mathrm{~s}^{2} 7 \mathrm{p}$ & ${ }^{1} \mathrm{P}$ & 0.007 & 18.791 \\
\hline A8 & $2 s^{2}$ & ${ }^{1} \mathrm{~S}$ & $1 \mathrm{~s} 2 \mathrm{~s}^{2} 8 \mathrm{p}$ & ${ }^{1} \mathrm{P}$ & 0.005 & 18.742 \\
\hline A9 & $2 s^{2}$ & ${ }^{1} \mathrm{~S}$ & $1 \mathrm{~s} 2 \mathrm{~s}^{2} 9 \mathrm{p}$ & ${ }^{1} \mathrm{P}$ & 0.004 & 18.708 \\
\hline B1 & $2 s 2 p$ & ${ }^{3} \mathrm{P}$ & $1 \mathrm{~s} 2 \mathrm{~s} 2 \mathrm{p}^{2}$ & $\left({ }^{3} \mathrm{~S}\right)^{3} \mathrm{P}$ & 0.328 & 22.466 \\
\hline B2 & $2 \mathrm{~s} 2 \mathrm{p}$ & ${ }^{3} \mathrm{P}$ & $1 \mathrm{~s} 2 \mathrm{~s} 2 \mathrm{p}^{2}$ & $\left({ }^{3} S\right)^{3} \mathrm{D}$ & 0.178 & 22.431 \\
\hline B3 & $2 \mathrm{~s} 2 \mathrm{p}$ & ${ }^{3} \mathrm{P}$ & $1 \mathrm{~s} 2 \mathrm{~s} 2 \mathrm{p}^{2}$ & $\left({ }^{3} S\right)^{3} S$ & 0.039 & 22.239 \\
\hline B4 & $2 \mathrm{~s} 2 \mathrm{p}$ & ${ }^{3} \mathrm{P}$ & $1 \mathrm{~s} 2 \mathrm{~s} 2 \mathrm{p}^{2}$ & $\left({ }^{1} \mathrm{~S}\right)^{3} \mathrm{P}$ & 0.014 & 22.132 \\
\hline $\mathrm{C} 1$ & $2 s 2 p$ & ${ }^{1} \mathrm{P}$ & $1 \mathrm{~s} 2 \mathrm{~s} 2 \mathrm{p}^{2}$ & $\left({ }^{1} \mathrm{~S}\right)^{1} \mathrm{D}$ & 0.182 & 22.558 \\
\hline $\mathrm{C} 2$ & $2 s 2 p$ & ${ }^{1} \mathrm{P}$ & $1 \mathrm{~s} 2 \mathrm{~s} 2 \mathrm{p}^{2}$ & $\left({ }^{3} \mathrm{~S}\right){ }^{1} \mathrm{P}$ & 0.339 & 22.389 \\
\hline $\mathrm{C} 3$ & $2 \mathrm{~s} 2 \mathrm{p}$ & ${ }^{1} \mathrm{P}$ & $1 \mathrm{~s} 2 \mathrm{~s} 2 \mathrm{p}^{2}$ & $\left({ }^{1} S\right){ }^{1} S$ & 0.040 & 22.363 \\
\hline D1 & $2 p^{2}$ & ${ }^{3} \mathrm{P}$ & $1 \mathrm{~s} 2 \mathrm{~s}^{2} 2 \mathrm{p}$ & ${ }^{3} \mathrm{P}$ & 0.005 & 23.643 \\
\hline $\mathrm{D} 2$ & $2 p^{2}$ & ${ }^{3} \mathrm{P}$ & $1 \mathrm{~s} 2 \mathrm{p}^{3}$ & ${ }^{3} \mathrm{D}$ & 0.173 & 22.511 \\
\hline D3 & $2 p^{2}$ & ${ }^{3} \mathrm{P}$ & $1 \mathrm{~s} 2 \mathrm{p}^{3}$ & ${ }^{3} \mathrm{~S}$ & 0.151 & 22.474 \\
\hline D4 & $2 p^{2}$ & ${ }^{3} \mathrm{P}$ & $1 \mathrm{~s} 2 \mathrm{p}^{3}$ & ${ }^{3} \mathrm{P}$ & 0.105 & 22.339 \\
\hline E1 & $2 p^{2}$ & ${ }^{1} \mathrm{D}$ & $1 s 2 s^{2} 2 p$ & ${ }^{1} \mathrm{P}$ & 0.006 & 23.598 \\
\hline E2 & $2 p^{2}$ & ${ }^{1} \mathrm{D}$ & $1 \mathrm{~s} 2 \mathrm{p}^{3}$ & ${ }^{1} \mathrm{D}$ & 0.328 & 22.477 \\
\hline E3 & $2 p^{2}$ & ${ }^{1} \mathrm{D}$ & $1 \mathrm{~s} 2 \mathrm{p}^{3}$ & ${ }^{3} \mathrm{P}$ & 0.002 & 22.457 \\
\hline E4 & $2 p^{2}$ & ${ }^{1} \mathrm{D}$ & $1 \mathrm{~s} 2 \mathrm{p}^{3}$ & ${ }^{1} \mathrm{P}$ & 0.112 & 22.306 \\
\hline $\mathrm{F} 1$ & $2 p^{2}$ & ${ }^{1} \mathrm{~S}$ & $1 s 2 s^{2} 2 p$ & ${ }^{1} \mathrm{P}$ & 0.002 & 23.855 \\
\hline $\mathrm{F} 2$ & $2 p^{2}$ & ${ }^{1} \mathrm{~S}$ & $1 \mathrm{~s} 2 \mathrm{p}^{3}$ & ${ }^{1} \mathrm{P}$ & 0.438 & 22.536 \\
\hline
\end{tabular}

The lower levels $\left(1 \mathrm{~s}^{2} 2 \ell 2 \ell^{\prime}\right)$ of these transitions have accurately known energy levels $E_{i}$ (Wiese et al. 1996). For the upper levels ( $\left.1 \mathrm{~s} 2 \ell 2 \ell^{\prime} n \ell^{\prime \prime}\right)$ accurate energy levels $E_{j}$ can be obtained indirectly from Auger electron spectra through the relation $E_{j}=E_{\mathrm{A}}+I$, with $I$ the ionisation potential of $\mathrm{O} \mathrm{v}$, for 
Table 3. Core-excited energy levels in O v. Columns (1) and (2): configuration and term; Cols. (3) and (4): calculated energy of the Auger electron $E_{\mathrm{A}}$ for a transition to the $1 \mathrm{~s}^{2} 2 \mathrm{~s}{ }^{2} \mathrm{~S}$ (ground) state of $\mathrm{O} \mathrm{VI}$ where only transition energies to the $1 \mathrm{~s}^{2} 2 \mathrm{p}{ }^{2} \mathrm{P}$ state are given in the original reference these have been corrected for the energy difference between the ${ }^{2} \mathrm{~S}$ and ${ }^{2} \mathrm{P}$ state; Col. (5): measured $E_{\mathrm{A}}$; Col. (6): adopted $E_{\mathrm{A}}$; Col. (7): adopted energy $E_{j}=E_{\mathrm{A}}+I$ of the level.

\begin{tabular}{|c|c|c|c|c|c|c|}
\hline Conf. & Term. & $\begin{array}{l}E_{\mathrm{A}}(\text { th) } \\
(\mathrm{eV})\end{array}$ & $\begin{array}{l}E_{\mathrm{A}}(\mathrm{th}) \\
(\mathrm{eV})\end{array}$ & $\begin{array}{l}E_{\mathrm{A}}(\exp ) \\
(\mathrm{eV})\end{array}$ & $\begin{array}{l}E_{\mathrm{A}} \\
(\mathrm{eV})\end{array}$ & $\begin{array}{l}E_{j} \\
(\mathrm{eV})\end{array}$ \\
\hline \multirow{2}{*}{$1 \mathrm{~s} 2 \mathrm{~s}^{2} 2 \mathrm{p}$} & & $441.16^{a}$ & $440.55^{d}$ & $440.5 \pm 0.2^{f}$ & 440.55 & $554.45 \pm 0.20$ \\
\hline & ${ }^{3} \mathrm{P}$ & $436.63^{a}$ & $435.96^{b}$ & $435.9 \pm 0.2^{f}$ & 435.96 & $549.86 \pm 0.20$ \\
\hline \multicolumn{2}{|c|}{$1 \mathrm{~s} 2 \mathrm{~s}^{2} 3 \mathrm{p}^{1} \mathrm{P}$} & & $507.54^{d}$ & & 507.54 & $621.44 \pm 0.20$ \\
\hline \multirow[t]{8}{*}{$1 \mathrm{~s} 2 \mathrm{~s} 2 \mathrm{p}^{2}$} & & $462.10^{a}$ & $460.83^{c}$ & $460 \pm 2^{e}$ & 460.83 & $574.73 \pm 0.20$ \\
\hline & ${ }^{1} \mathrm{P}$ & $461.98^{a}$ & $460.12^{c}$ & $460.5 \pm 1^{e}$ & 460.12 & $574.02 \pm 0.20$ \\
\hline & ${ }^{1} \mathrm{D}$ & $456.74^{a}$ & $455.35^{c}$ & $456.3 \pm 0.8^{e}$ & 455.35 & $569.25 \pm 0.20$ \\
\hline & ${ }^{3} \mathrm{~S}$ & $455.06^{a}$ & $454.17^{b}$ & $454 \pm 1^{e}$ & 454.17 & $568.07 \pm 0.20$ \\
\hline & $\left({ }^{3} \mathrm{~S}\right)^{3} \mathrm{P}$ & $448.76^{a}$ & $448.70^{b}$ & $448.6 \pm 0.2^{f}$ & 448.70 & $562.60 \pm 0.20$ \\
\hline & $\left({ }^{1} \mathrm{~S}\right)^{3} \mathrm{P}$ & $458.58^{a}$ & $456.9^{e}$ & & 457.50 & $571.40 \pm 0.60$ \\
\hline & ${ }^{3} \mathrm{D}$ & $449.74^{a}$ & $448.68^{b}$ & $448.5 \pm 1^{e}$ & 448.68 & $562.58 \pm 0.20$ \\
\hline & ${ }^{5} \mathrm{P}$ & $438.33^{a}$ & & $438.55 \pm 0.2^{f}$ & 438.55 & $552.45 \pm 0.20$ \\
\hline \multirow[t]{6}{*}{$1 \mathrm{~s} 2 \mathrm{p}^{3}$} & ${ }^{1} \mathrm{P}$ & $473.72^{a}$ & $471.80^{d}$ & & 471.80 & $585.70 \pm 0.20$ \\
\hline & ${ }^{1} \mathrm{D}$ & $468.05^{a}$ & & & 466.97 & $580.87 \pm 0.60$ \\
\hline & ${ }^{3} \mathrm{~S}$ & $464.91^{a}$ & & & 463.83 & $577.73 \pm 0.60$ \\
\hline & ${ }^{3} \mathrm{P}$ & $469.40^{a}$ & & & 468.32 & $582.22 \pm 0.60$ \\
\hline & ${ }^{3} \mathrm{D}$ & $463.75^{a}$ & & & 462.67 & $576.57 \pm 0.60$ \\
\hline & ${ }^{5} \mathrm{~S}$ & $456.27^{a}$ & & $456.4 \pm 0.2^{f}$ & 456.40 & $570.30 \pm 0.20$ \\
\hline
\end{tabular}

a Chen (1985).

${ }^{b}$ Lin et al. (2001).

$c$ Shiu et al. (2001).

$d$ Lin et al. (2002).

$e$ Bruch et al. (1979).

$f$ Bruch et al. (1987).

which we take $113.896 \mathrm{eV}$ (Wiese et al. 1996), and $E_{\mathrm{A}}$ the energy of the Auger electron.

$E_{\mathrm{A}}$ has been measured in the laboratory or calculated theoretically; we list the relevant values in Table 3. A comparison of the calculations of the group of K.T. Chung (Lin et al. 2001; Shiu et al. 2001; Lin et al. 2002) with the measurements of Bruch et al. $(1979,1987)$ shows excellent agreement; the average difference between observed and calculated values is $-0.05 \pm 0.16 \mathrm{eV}$; we therefore adopt a nominal uncertainty of $0.20 \mathrm{eV}$ for these calculated energies, equal to the measurement errors in the best measurements. A comparison of the energies calculated by Chen (1985) with the above mentioned calculations by the group of K.T. Chung shows an average difference of $1.08 \pm 0.28 \mathrm{eV}$, with a standard deviation of $0.60 \mathrm{eV}$ for the 9 levels in common. Therefore whenever we use the energies calculated by Chen, we subtract $1.08 \mathrm{eV}$ and assign a nominal accuracy of $0.60 \mathrm{eV}$.

The adopted energies $E_{j}$ of Table 3 were combined with the lower level energies $E_{i}$ of Table 1 in order to calculate the predicted wavelengths of the transitions from Table 2 . We list those wavelengths in Table 4. Champeaux et al. (2003) recently deduced the ionisation potential $I$ for $\mathrm{OV}$ to be $113.66 \pm 0.13 \mathrm{eV}$, based upon the quantum defect estimated
Table 4. Wavelength comparison of the X-ray lines of OV. Wavelengths are given in $\AA$. The column "Cowan" gives $\lambda$ from Table 2; "corr." are these calculated wavelengths corrected as described in the text; "Auger" are the wavelengths determined from Auger energies as listed in Table 3; "CC" are the calculations by Chen \& Crasemann (1987); "HULLAC" are the wavelengths calculated using the HULLAC code.

\begin{tabular}{llllll}
\hline \hline Line & Cowan & Corr. & Auger & CC & HULLAC \\
\hline A2 & 22.381 & 22.374 & $22.362 \pm 0.008$ & 22.415 & 22.372 \\
A3 & 19.871 & 19.866 & $19.951 \pm 0.008$ & & 19.921 \\
A4 & 19.258 & 19.253 & & & 19.316 \\
A5 & 19.002 & 18.997 & & & 19.060 \\
A6 & 18.869 & 18.864 & & & 18.949 \\
A7 & 18.791 & 18.786 & & & 18.849 \\
A8 & 18.742 & 18.737 & & & 18.800 \\
A9 & 18.708 & 18.703 & & & 18.766 \\
B1 & 22.466 & 22.488 & $22.444 \pm 0.008$ & 22.535 & 22.425 \\
B2 & 22.431 & 22.453 & $22.445 \pm 0.008$ & 22.494 & 22.415 \\
B3 & 22.239 & 22.260 & $22.224 \pm 0.008$ & 22.279 & 22.197 \\
B4 & 22.132 & 22.153 & $22.092 \pm 0.024$ & 22.139 & 22.091 \\
C1 & 22.558 & 22.589 & $22.561 \pm 0.008$ & 22.668 & 22.522 \\
C2 & 22.389 & 22.419 & $22.367 \pm 0.008$ & 22.453 & 22.365 \\
C3 & 22.363 & 22.393 & $22.338 \pm 0.008$ & 22.448 & 22.349 \\
D1 & 23.643 & 23.670 & $23.690 \pm 0.009$ & 23.790 & 23.572 \\
D2 & 22.511 & 22.538 & $22.540 \pm 0.024$ & 22.615 & 22.478 \\
D3 & 22.474 & 22.501 & $22.492 \pm 0.024$ & 22.566 & 22.404 \\
D4 & 22.339 & 22.365 & $22.311 \pm 0.024$ & 22.383 & 22.331 \\
E1 & 23.598 & 23.597 & $23.584 \pm 0.009$ & 23.747 & 23.520 \\
E2 & 22.477 & 22.476 & $22.455 \pm 0.024$ & 22.584 & 22.430 \\
E3 & 22.457 & 22.456 & $22.400 \pm 0.024$ & 22.529 & 22.479 \\
E4 & 22.306 & 22.305 & $22.260 \pm 0.008$ & 22.353 & 22.283 \\
F1 & 23.855 & 23.908 & $23.901 \pm 0.009$ & 24.112 & 23.765 \\
F2 & 22.536 & 22.589 & $22.543 \pm 0.008$ & 22.677 & 22.503 \\
\hline & & & & &
\end{tabular}

from resonances in the measured photoionisation cross section. If this value is used instead of the value of $113.896 \mathrm{eV}$ given by Wiese et al. (1996), most wavelengths listed in the column “Auger" of Table 4 increase by $0.009 \AA$.

A comparison of the energies of the $1 s^{2} 2 \ell 2 \ell^{\prime}$ levels calculated by us using the Cowan (1981) code with the values of Table 1 (Wiese et al. 1996) shows that our calculated energies are smaller by 0.71 (levels 2-4), 0.91 (level 5), 0.83 (levels 6-8), 0.16 (level 9) and $1.46 \mathrm{eV}$ (level 10), respectively. Correcting all wavelengths where these levels are involved by these differences and also adjusting all K-shell vacancy states by the same number so as to get the laboratory wavelength of the $22.374 \AA$ A line correct, yields the column labeled with "corr." in Table 4. The difference in wavelength between both columns can be as large as $0.053 \AA$, comparable to the energy resolution of Chandra's LETGS. Finally, we list in Table 4 also wavelengths from the paper by Chen \& Crasemann (1987) as well as wavelengths presently calculated with the Hebrew University Lawrence Livermore Atomic Code (HULLAC).

In general, the theoretical calculations for the wavelengths differ quite a lot. We illustrate this with the resonance line at 22.374 A. Calculated values range from $22.362 \AA$ (Auger measurements) to $22.415 \AA$ (Chen \& Crasemann); for comparison, Pradhan et al. (2003) give a value of $22.35 \AA$. 
Table 5. Oscillator strengths $f$, total transition probabilities $A_{\text {rad, }}$, Auger decay rates $A_{\mathrm{A}}$ and total transition rates $A_{\mathrm{tot}}$ of $\mathrm{OV}$. Transition probabilities are in units of $10^{12} \mathrm{~s}^{-1}$.

\begin{tabular}{|c|c|c|c|c|c|c|}
\hline id & $f^{a}$ & $\overline{f^{b}}$ & $A_{\mathrm{rad}}{ }^{a}$ & $\overline{A_{\mathrm{rad}}{ }^{b}}$ & $A_{\mathrm{A}}^{a, c}$ & $\overline{A_{\mathrm{tot}}{ }^{d}}$ \\
\hline A2 & 0.646 & 0.649 & 3.05 & 3.00 & 87.9 & 90.9 \\
\hline A 3 & & 0.108 & & 0.63 & 129 & 129.6 \\
\hline A4 & & 0.041 & & 0.26 & 129 & 129.3 \\
\hline A5 & & 0.020 & & 0.13 & 129 & 129.1 \\
\hline A6 & & 0.012 & & 0.07 & 129 & 129.1 \\
\hline A7 & & 0.007 & & 0.05 & 129 & 129.1 \\
\hline A8 & & 0.005 & & 0.03 & 129 & 129.0 \\
\hline A9 & & 0.004 & & 0.02 & 129 & 129.0 \\
\hline B1 & 0.326 & 0.328 & 4.29 & 4.34 & 34.2 & 38.5 \\
\hline B2 & 0.171 & 0.178 & 1.35 & 1.41 & 94.2 & 95.6 \\
\hline B3 & 0.039 & 0.039 & 1.56 & 1.56 & 46.9 & 48.5 \\
\hline B4 & 0.014 & 0.014 & 0.19 & 0.19 & 100 & 100.2 \\
\hline $\mathrm{C} 1$ & 0.181 & 0.182 & 1.41 & 1.43 & 213 & 214.4 \\
\hline $\mathrm{C} 2$ & 0.333 & 0.339 & 4.40 & 4.51 & 22.9 & 27.4 \\
\hline C3 & 0.041 & 0.040 & 1.63 & 1.60 & 154 & 155.6 \\
\hline D1 & 0.007 & 0.005 & 0.08 & 0.06 & 129 & 129.1 \\
\hline D2 & 0.169 & 0.173 & 1.32 & 1.37 & 131 & 132.4 \\
\hline D3 & 0.153 & 0.151 & 6.00 & 5.99 & 0.0015 & 6.0 \\
\hline D4 & 0.102 & 0.105 & 1.36 & 1.42 & 79.9 & 81.3 \\
\hline E1 & 0.009 & 0.006 & 3.05 & 3.00 & 87.9 & 90.9 \\
\hline E2 & 0.330 & 0.328 & 4.31 & 4.35 & 130 & 134.4 \\
\hline E3 & 0.000 & 0.002 & 1.36 & 1.42 & 79.9 & 81.3 \\
\hline E4 & 0.111 & 0.112 & 4.36 & 4.41 & 76.9 & 81.3 \\
\hline F1 & 0.003 & 0.002 & 3.05 & 3.00 & 87.9 & 90.9 \\
\hline F2 & 0.439 & 0.438 & 4.36 & 4.41 & 76.9 & 81.3 \\
\hline
\end{tabular}

a Chen \& Crasemann (1987).

$b$ Present work.

c For A3-A9 see text.

${ }^{d}$ Using $A_{\mathrm{tot}}=A_{\text {rad }}+A_{\mathrm{A}}$ with $A_{\text {rad }}$ from the present work.

There is a good agreement between our calculations for oscillator strengths and transition probabilities and those of Chen \& Crasemann (1987), see Table 5. The rms difference between both sets of oscillator strength is 0.003 . Also, the total radiative transition probabilities from core-excited levels agree within $2 \%$. It is necessary to take also the Auger transitions into account for estimating the lifetime of the core-excited levels. We use the Auger rates determined by Chen \& Crasemann (1987). Their rates are in good agreement with the calculations of Lin et al. $(2001,2002)$ for the 6 levels in common. These Auger rates are an order of magnitude larger than the radiative transition rates. Thus neglecting Auger rates will affect the estimated line equivalent width for high column densities. There are no Auger rates available for the A4-A9 transitions. Lin et al. (2002) calculate an Auger rate of $1.29 \times 10^{14} \mathrm{~s}^{-1}$ for the upper level of transition A3. In their calculations for Be, they find that for higher principal quantum number $n$ the Auger rates do not depend strongly upon $n$. Therefore we adopt a constant value for $A_{\mathrm{A}}$ for transitions A3-A9.

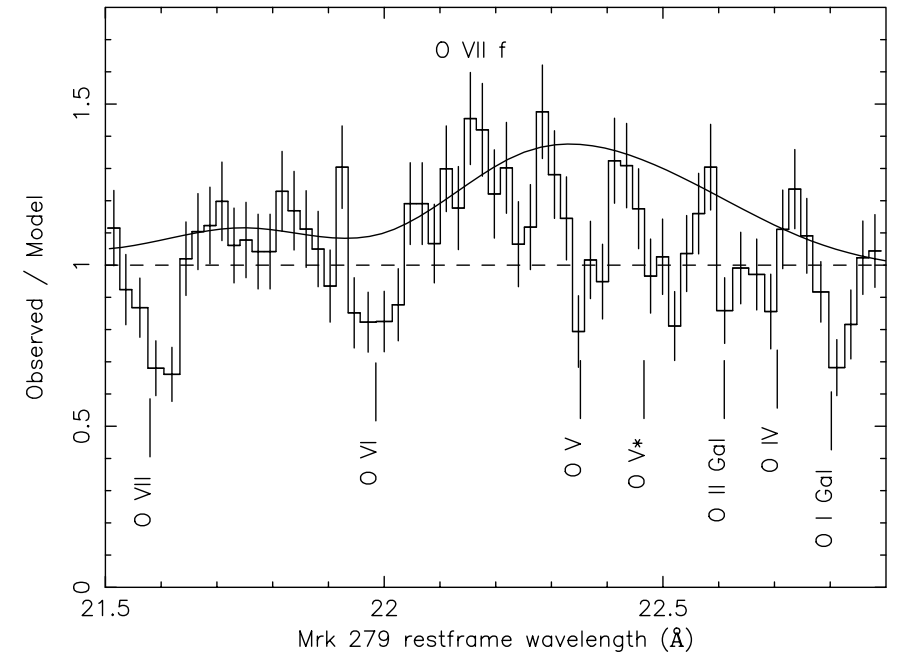

Fig. 7. Chandra LETGS spectrum of Mrk 279 near the oxygen absorption lines. The observed spectrum has been divided by the best fit simple continuum model described in the text (dashed line). The additional broad emission feature is indicated by the curved line. The wavelength scale is for the restframe of Mrk 279 (at $z=0.0306$ ). In the line identifications, we have taken into account a small outflow (blueshift) of $300 \mathrm{~km} \mathrm{~s}^{-1}$ with respect to this restframe; this outflow has been measured using simultaneous high-resolution UV spectra. A few Galactic absorption lines are labeled with "Gal".

\section{Comparison with the observed spectrum of Mrk 279}

\subsection{Chandra LETGS observations}

Mrk 279 is a moderately distant $(z=0.0306)$ Seyfert 1 galaxy that we observed for $340 \mathrm{ks}$ with the Chandra LETGS. This instrument has a spectral resolution of $\sim 0.05 \AA F W H M$. The full data analysis of the LETGS spectrum of Mrk 279 will be presented elsewhere (Costantini et al. 2004). Here we only summarize the results relevant for the study of the $\mathrm{O} v$ absorption lines. The LETGS spectrum was fit by a simple power law plus modified blackbody with Galactic absorption. This smooth continuum spectrum produces an excellent fit to the data except for the few regions where Mrk 279 has absorption lines. The column densities in Mrk 279 are several times smaller than in other well-studied AGN, and therefore most absorption lines and edges are very weak except for the lines from the most abundant metal, which is oxygen. These lines are predominantly found in the 18-24 A band.

Our continuum fit is excellent everywhere except for the region near the K-shell transitions of oxygen (Fig. 7). Here a broad emission feature with a peak amplitude of about $30 \%$ of the continuum is visible. Similar broad X-ray emission lines have been seen before in other AGN (Kaastra et al. 2002; Steenbrugge et al. 2004), and may have asymmetric line profiles. This broad line is due to the O VII triplet from the broad line region. The presence of this broad feature makes the estimate of the underlying continuum for the narrow absorption lines less certain. We estimated the broad line profile by drawing a spline through the spectral regions that are known to be line-free. 
Table 6. Observed absorption lines in Mrk 279, due to or related to $\mathrm{OV}$. The observed wavelength $\lambda$ is given with two error bars: the first is the statistical uncertainty, the second the systematic due to the wavelength calibration uncertainty of the LETGS. For lines with wavelength fixed, the observed equivalent width $(E W)$ is merely the formal upper limit. The line around $22.50 \AA$ is either fitted by a single line $(22.540 \AA)$ or alternatively by two lines (22.501 and $22.548 \AA$ ).

\begin{tabular}{lll}
\hline \hline$\lambda(\AA)$ & $E W(\mathrm{~m} \AA)$ & Identification \\
\hline $22.377 \pm 0.009 \pm 0.008$ & $18 \pm 6$ & $\mathrm{~A} 2$ \\
19.957 (fixed) & $2 \pm 8$ & $\mathrm{~A} 3$ \\
19.325 (fixed) & $6 \pm 6$ & $\mathrm{~A} 4$ \\
$22.540 \pm 0.009 \pm 0.010$ & $20 \pm 5$ & $\mathrm{~B} 1+\mathrm{B} 2 ? ;$ see text \\
$22.501 \pm 0.016 \pm 0.010$ & $10 \pm 6$ & $\mathrm{~B} 1+\mathrm{B} 2 ? ;$ see text \\
$22.548 \pm 0.010 \pm 0.010$ & $18 \pm 6$ & $? ;$ see text \\
$22.269 \pm 0.015 \pm 0.006$ & $10 \pm 6$ & $\mathrm{~B} 3 ? ;$ see text \\
\hline
\end{tabular}

From a detailed study of the OVI UV and X-ray lines (Gabel et al. 2004b; Costantini et al. 2004) we find that the dominant absorber has an outflow velocity of $300 \mathrm{~km} \mathrm{~s}^{-1}$ and a line profile that can be approximated to first order by a Gaussian with $\sigma_{\mathrm{v}}=50 \mathrm{~km} \mathrm{~s}^{-1}$.

\subsection{Observed Ov lines}

We clearly see the absorption at $22.37 \AA$, which we identify as the $1 s^{2} 2 s^{2}-1 s 2 s^{2} 2 p{ }^{1} P_{1}$ transition of $\mathrm{OV}$ (Fig. 7). Its measured wavelength in the rest frame of Mrk 279, corrected for the $300 \mathrm{~km} \mathrm{~s}^{-1}$ outflow velocity, is $22.377 \pm 0.009 \AA$, in excellent agreement with the laboratory wavelength of $22.374 \AA$ mentioned before (Schmidt et al. 2004). The quoted error bar is only the statistical error; we estimate the systematic uncertainty (due to calibration uncertainties in the HRC-S detector) to be $0.008 \AA$.

We find no clear evidence for transition A3. Upper limits to its equivalent width depend upon the adopted wavelength. In another Seyfert 1 galaxy with a strong A2 line we find evidence for a $2 \sigma$ detection of transition A3 at a laboratory wavelength of $19.957 \pm 0.015 \AA$, right within the wavelength range indicated in Table 4 . We adopt this wavelength for transition A3 and use it to measure the limits to the line equivalent width (Table 6). We also do not detect line A4. We take here the wavelength as calculated with the HULLAC code to estimate the equivalent width. Using the velocity broadening $\sigma_{v}$ of $50 \mathrm{~km} \mathrm{~s}^{-1}$ and the equivalent widths of lines A2, A3 and A4, we find a column density of $10^{20.16 \pm 0.42} \mathrm{~m}^{-2}$ for $\mathrm{OV}$ in its ground state. The large uncertainty is mainly due to the strong saturation of the A2 line, which has an optical depth of 3.7 at its line center.

Most absorption lines in the spectral band displayed in Fig. 7 can be easily identified by Galactic absorption lines or absorption lines due to the warm absorber. Only the feature around $22.50 \AA$ has no obvious identification with absorption from the ground state of any abundant ion, either in the warm absorber or the Galactic foreground. When fitted by a single line, its laboratory wavelength, corrected for the

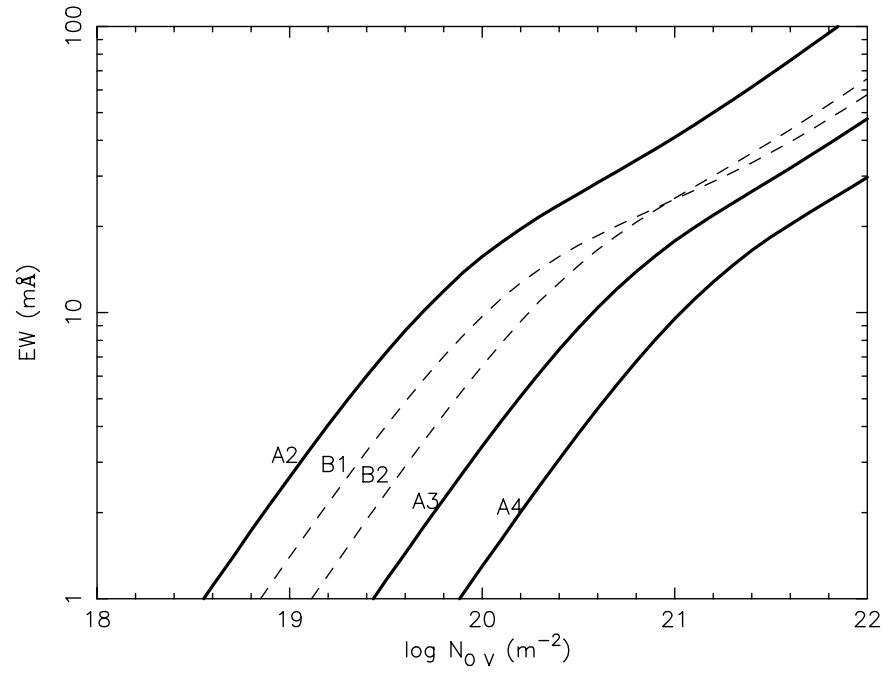

Fig. 8. Curve of growth for the most important K-shell absorption lines of $\mathrm{Ov}$, for a Gaussian velocity broadening $\sigma_{v}$ of $50 \mathrm{~km} \mathrm{~s}^{-1}$.

$300 \mathrm{~km} \mathrm{~s}^{-1}$ outflow, is $22.540 \pm 0.009 \AA$. A marginally better fit $\left(\Delta \chi^{2}=-3.6\right.$ for two additional degrees of freedom) is obtained for a double, blended line, with laboratory wavelengths of $22.501 \pm 0.016 \AA$ and $22.548 \pm 0.010 \AA$, respectively (see Table 6). Again, quoted errors are statistical only; the systematic uncertainty is $0.010 \AA$ and is not correlated with the systematic uncertainty in the main $22.374 \AA$ line.

We suggest that in the case of a double blended line, the $22.501 \AA$ component of the blend may be due to a combination of the $\mathrm{B} 1$ and $\mathrm{B} 2$ lines of $\mathrm{OV}$, which have a wavelength in the

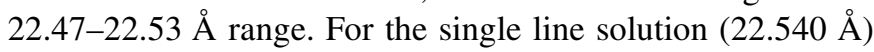
this is somewhat less likely. We note that the added statistical and systematic uncertainty of the measurement of the line centroid is in the $0.02-0.03 \AA$ A range. The problem with a firm identification lies of course in both the uncertainty in the theoretical wavelength as well as in the measured wavelength.

If our identification is true, we can deduce the column density of $\mathrm{OV}$ in its $2 \mathrm{~s} 2 \mathrm{p}^{3} \mathrm{P}$ triplet state. From the curve of growth (see Fig. 8) and an equivalent width of $10 \pm 6 \mathrm{~m} \AA$, we find a column of $10^{19.82 \pm 0.44} \mathrm{~m}^{-2}$. This combined with the column density of $\mathrm{OV}$ in its ground state yields a value for the population of the $2 \mathrm{~s} 2 \mathrm{p}{ }^{3} \mathrm{P}$ triplet state relative to the ground state of 0.5 , with rms error bounds of a factor of 4 (i.e., the relative population is between 0.125 and 2). From the column density of the $2 \mathrm{~s} 2 \mathrm{p}^{3} \mathrm{P}$ triplet state we predict that line $\mathrm{B} 3$ should have an equivalent width of $1 \mathrm{~m} \AA$ with an upper limit of $3 \mathrm{~m} \AA$. The feature at $22.269 \AA$ (see Table 6) could be marginally consistent with this.

Table 7 list the densities derived from the population ratio for a few temperatures.

From our photoionisation equilibrium calculations, it follows that $\mathrm{OV}$ has its maximum concentration for $k T=2 \mathrm{eV}$ (see Fig. 4). This yields a lower limit to the density of $2.5 \times$ $10^{18} \mathrm{~m}^{-3} \cdot k T=2 \mathrm{eV}$ corresponds to $\xi=0.9 \times 10^{-9} \mathrm{~W} \mathrm{~m}^{-2} \mathrm{~m}^{3}$, which then leads to an upper limit to the distance $r$ of $2 \times 10^{14} \mathrm{~m}$ or one light week. This would place the $\mathrm{O} \mathrm{V}$ absorber close to the inner broad line region. Although not completely impossible, such a small radius is not very plausible, because Balmer 
Table 7. Densities (in $\mathrm{m}^{-3}$ ) for $2 \mathrm{~s} 2 \mathrm{p}$ triplet to ground ratio's of 0.125 , 0.5 and 2 , for a total $\mathrm{O} v$ column density of $1.5 \times 10^{20} \mathrm{~m}^{-2}$.

\begin{tabular}{llll}
\hline \hline$k T(\mathrm{eV})$ & 0.125 & 0.5 & 2 \\
\hline 2 & $2.5 \times 10^{18}$ & $3 \times 10^{19}$ & $\infty$ \\
4 & $10^{17}$ & $10^{18}$ & $1.5 \times 10^{19}$ \\
6 & $5 \times 10^{16}$ & $4 \times 10^{17}$ & $10^{19}$ \\
\hline
\end{tabular}

line time-delay measurements suggest a broad emission line region size of 6-17 light days for Mrk 279 (Scott et al. 2004, and references therein), and UV spectra show that the broad lines are covered by the warm absorber.

The temperature may be higher than $2 \mathrm{eV}$, however, if additional heating processes occur in the absorbing region. Cloudy (Ferland 2002) allows for the inclusion of additional heat sources. A temperature of $6 \mathrm{eV}$ is reached for $\xi=$ $0.7 \times 10^{-9} \mathrm{~W} \mathrm{~m}^{-2} \mathrm{~m}^{3}$, with an additional heating rate of $1.0 \times$ $10^{-7} n_{14}^{2} \mathrm{~W} \mathrm{~m}^{-3}$, where $n_{14}$ the density in units of $10^{14} \mathrm{~m}^{-3}$. In this case the additional heat source dominates the total heating rate. This is also the case for all temperatures above $3 \mathrm{eV}$, where the additional heating contributes more than $80 \%$ to the total heating rate. We find for $k T=6 \mathrm{eV}$ a lower limit to the density of $5 \times 10^{16} \mathrm{~m}^{-3}$, corresponding to an upper limit to the distance of $1.5 \times 10^{15} \mathrm{~m}$ or 59 light days. This would place the absorber outside the broad line region.

Is there a possible heating process that can produce the additional heat? For the lower limit to the density of $5 \times 10^{16} \mathrm{~m}^{-3}$, the required heating rate $Q$ is $0.025 \mathrm{~W} \mathrm{~m}^{-3}$. One possibility would be classical heat conduction from a neighbouring hot region. Using a hydrogen column density of $8 \times 10^{23} \mathrm{~m}^{-2}$ (corresponding to a typical $\mathrm{O} \mathrm{V}$ column of $1.5 \times 10^{20} \mathrm{~m}^{-2}$ ) and the lower limit of $5 \times 10^{16} \mathrm{~m}^{-3}$ for the density, we find an upper limit to the size $d$ of the absorber of $1.6 \times 10^{7} \mathrm{~m}$. For a heat conductivity $\kappa$ of $10^{-10} T_{\mathrm{K}}^{5 / 2} \mathrm{~W} \mathrm{~K}^{-1} \mathrm{~m}^{-1}$ with $T_{\mathrm{K}}$ the temperature in $\mathrm{K}$, and putting the heating rate $Q$ due to conduction equal to $\kappa T / d^{2}$, we find that we need a temperature of $300 \mathrm{eV}$ for the hot gas surrounding the absorbing medium. Hotter gas in photoionisation equilibrium coexisting with colder gas is indeed known to be possible. As the geometry of the absorbing regions is still not fully understood, we leave this scenario as an interesting possibility.

In our above analysis, we assumed that as far as $\mathrm{OV}$ is concerned the physical conditions in the absorber are such that $\mathrm{OV}$ has its maximum relative concentration. It is of course also possible to have a hotter absorber with a smaller concentration of $\mathrm{O} v$. For instance, without invoking an additional heat source we find that $k T=4 \mathrm{eV}$ if $\xi$ is 20 times larger than we used before, and correspondingly the $\mathrm{OV} / \mathrm{O}$ ratio is 100 times smaller. In that case most of the oxygen should be in the form of O VII. Our column densities for O VI and O VII (see Costantini et al. 2004) are not inconsistent with such a scenario. In this case, we avoid the additional heating problem, but of course we still require a high density $n>10^{17} \mathrm{~m}^{-3}$ in order to get the high metastable population fraction. In this case however the distance to the central source is only $2 \times 10^{14} \mathrm{~m}$ or 8 light days.

We do not expect blending due to lines from higher levels to be important for all temperatures below $4 \mathrm{eV}$ (the line series labeled with $\mathrm{C}-\mathrm{F}$ of Table 2). This is because for those temperatures the population of these higher levels is small.

Finally, if the metastable level has a significant population, also the UV absorption lines from this level should be detectable in principle. Unfortunately the strong, allowed UV absorption lines from the metastable level are in the unobserved part of the UV spectrum (near $760 \AA$ ). If the density would be very high such that also the $2 \mathrm{~s} 2 \mathrm{p}^{1} \mathrm{P}_{1}$ level gets a high population, then the strongest UV absorption line should be the transition between levels 5-9 of Table 1 at $1371.30 \AA$ A. Simultaneous HST observations of Mrk 279 (Gabel et al. 2004b) yield an upper limit to the column density for level 5 deduced from the non-detection of this line of $5 \times 10^{16} \mathrm{~m}^{-2}$. Combined with the allowed range for the ground state column density $\left(10^{19.82 \pm 0.44} \mathrm{~m}^{-2}\right.$ as quoted before $)$ this leads to an upper limit of $10^{-3}$ for the population of level 5 with respect to the ground. The corresponding upper limit to the density of $5 \times 10^{17} \mathrm{~m}^{-3}$ is consistent with the lower limits derived from the X-ray transition from the metastable level. It should be noted, however, that the X-ray derived density constraints depend on several modeling and observational factors, as is evident from our present discussion.

\section{Conclusions}

We have investigated in this paper the population of the metastable level of $\mathrm{O} v$ under photoionised conditions. For sufficiently high densities and a temperature of just a few eV the metastable levels can have a significant population. The relevant critical densities also depend strongly upon the column density, due to resonance line trapping. The population of higher levels than the $2 \mathrm{~s} 2 \mathrm{p}{ }^{3} \mathrm{P}$ triplet is always relatively small. If the metastable level has a significant population, then we should observe K-shell absorption lines from these levels. The wavelengths of these lines are not very accurately known, however. This makes the identification of these lines in the X-ray spectrum of the Seyfert 1 galaxy Mrk 279 somewhat uncertain. If present, however, then the observed equivalent widths imply densities of order $10^{17} \mathrm{~m}^{-3}$ or more, corresponding to a distance to the central source of the order of a light month.

Acknowledgements. Unfortunately, Rolf Mewe died unexpectedly just a week before the first draft of this paper was finished. His broad overview and long standing record in the field of X-ray spectroscopy, as well as his friendship will not be forgotten by us. The Space Research Organization of the Netherlands is supported financially by NWO, the Netherlands Organization for Scientific Research. N.A. aknowledges NASA grants HST-GO-9688 and Chandra-04700532. E.B. was supported by the Yigal-Alon Fellowship and by ISF grant \#28/03.

\section{References}

Arav, N., Korista, K. T., de Kool, M., Junkkarinen, V. T., \& Begelman, M. C. 1999, ApJ, 516, 27

Arnaud, M., \& Rothenflug, R. 1985, A\&AS, 60, 425

Behar, E., Rasmussen, A. P., Blustin, A. J., et al. 2003, ApJ, 598, 232

Bhatia, A. K., \& Kastner, S. O. 1993, ApJ, 408, 744

Bruch, R., Schneider, D., Schwarz, W. H. E., et al. 1979, Phys. Rev. A, 19, 587 
Bruch, R., Stolterfoht, N., Datz, S., et al. 1987, Phys. Rev. A, 35, Lin, S.-H., Hsue, C.-S., \& Chung, K. T. 2001a, Phys. Rev. A, 64, 4114 12709

Champeaux, J.-P., Bizau, J.-M., Cubaynes, D., et al. 2003, ApJS, 148, Lin, S.-H., Hsue, C.-S., \& Chung, K. T. 2001b, Phys. Rev. A, 65, 583

Chen, M. H. 1985, Phys. Rev. A, 31, 1449

Chen, M. H., \& Crasemann, B. 1987, ADNDT, 37, 419

Costantini, E., Kaastra, J. S., Arav, N., et al. 2004, in preparation

Cowan, R. D. 1981, The theory of atomic structure and spectra (Berkeley: Univ. of California Press)

Crenshaw, D. M., Kraemer, S. B., Boggess, A., et al. 1999, ApJ, 516, 750

Doyle, J. G., Kingston, A. E., \& Reid, R. H. G. 1980, A\&A, 90, 97

Ferland, G. J. 2002, Hazy, a brief introduction to Cloudy 96, Univ. Kentucky Phys. and Astron. Dept. internal report

Gabel, J. R., Crenshaw, D. M., Kraemer, S. B., et al. 2003, ApJ, 583, 178

Gabel, J. R., Kraemer, S. B., \& Crenshaw, D. M. 2004a, in AGN physics with the SDSS, ASP Conf. Ser., 311, 239

Gabel, J. R., et al. 2004b, in preparation

George, I. M., Turner, T. J., Netzer, H., et al. 1998, ApJS, 114, 73

Hollenbach, D., \& McKee, C. F. 1979, ApJS, 41, 555

Kaastra, J. S., Mewe, R., Liedahl, D. A., Komossa, S., \& Brinkman, A. C. 2000, A\&A, 354, L83

Kaastra, J. S., Steenbrugge, K. C., Raassen, A. J. J., et al. 2002, A\&A, 386,427

Kallman, T. R., \& McCray, R. 1982, ApJS, 50, 263

Kato, T., Lang, J., \& Berrington, K. E. 1990, ADNDT, 44, 133

Korista, K., Weymann, R. J., Morris, S. L., et al. 1992, ApJ, 401, 529

Netzer, H., Kaspi, S., Behar, E., et al. 2003, ApJ, 599, 933

Ogle, P. M., Mason, K. O., Page, M. J., et al. 2004, ApJ, 606, 151

Pettini, M., \& Boksenberg, A. 1986, in New Insights in Astrophysics, Eight Years of UV Astronomy with IUE, ESA-SP 263, 627

Pradhan, A. K., Chen, G. X., Delahaye, F., Nahar, S. N., \& Oelgoetz, J. 2003, MNRAS, 341, 1268

Rakovic, M., Wang, J. G., Schultz, D. R., \& Stancil, P. C. 2001, ORNL/UGA Charge Transfer Database for Astrophysics, http://www-cfadc.phy.ornl.gov/astro/ps/data/

Reeves, J. N., Nandra, K., George, I. M., et al. 2004, ApJ, 602, 648

Reynolds, C. S. 1997, MNRAS, 286, 513

Safranova, U. I., Shlyaptseva, A. S., Kato, T., Masai, K., \& Vainshtein, L. A. 1995, ADNDT, 60, 1

Schmidt, M., Beiersdorfer, P., Chen, H., et al. 2004, ApJ, 604, 562

Scott, J. E., Kriss, G. A., Lee, J. C., et al. 2004, ApJS, 152, 1

Shiu, W. C., Hsue, C.-S., \& Chung, K. T. 2001, Phys. Rev. A, 64, 22714

Steenbrugge, K. C., Kaastra, J. S., de Vries, C. P., \& Edelson, R. 2003, A\&A, 402, 477

Steenbrugge, K. C., Kaastra, J. S., Crenshaw, D. M., et al. 2004, A\&A, submitted

Wiese, W. L., Fuhr, J. R., \& Deters, T. M. 1996, J. Phys. Chem. Ref. Data, Monograph 7

Zhang, H. L., \& Sampson, D. H. 1992, ADNDT, 52, 143 